\title{
Budd-Chiari syndrome - various etiologies and imagistic findings. A pictorial review
}

\section{Laura Iliescu, Letitia Toma, Adriana Mercan-Stanciu, Mihaela Grumeza, Mihai Dodot, Teodora Isac, Simona Ioanitescu}

Department of Internal Medicine, Fundeni Clinical Institute, Bucharest, Romania

\begin{abstract}
Budd Chiari syndrome defines an obstruction of the hepatic venous outflow. Primary causes include pro-coagulant states resulting in venous thrombosis, while secondary Budd Chiari syndrome appears in conditions associated with extrinsic compression of the hepatic veins or tumor invasion. Clinical presentation is greatly varied, from incidentally discovered asymptomatic thrombosis to fulminant liver failure due to hepatic congestion. Abdominal ultrasonography is the key diagnostic tool of Budd Chiari syndrome. This pictorial essay aims to show the ultrasonographic aspect of Budd-Chiari syndrome associated with other medical conditions (abdominal malignancy, hematologic disorders and abdominal surgery).

Keywords: Budd Chiari syndrome; malignancy; surgery; myeloproliferative syndrome
\end{abstract}

\section{Introduction}

Hepatic vein thrombosis was described first in 1845 and has been extensively studied both as a clue to an undiagnosed condition and as a cause of liver dysfunction. Prothrombotic factors are often found in patients with Budd Chiari syndrome (BCS) [1]. These include myeloproliferative disorders (the most frequent), Factor $\mathrm{V}$ Leyden mutation, genetic deficit of protein $\mathrm{C}$ and $\mathrm{S}$, hormonal factors (such as pregnancy and the use of oral contraceptives), but also local factors such as diseases associated with abdominal inflammation (inflammatory bowel disease) or intra-abdominal neoplasms.

Two or more hepatic veins must be occluded for the disease to become clinically manifest [2]. As a result of

Received 15.02.2019 Accepted 15.03.2019

Med Ultrason

2019, Vol. 21, No 3, 344-348

Corresponding author: Laura Iliescu, MD

Department of Internal Medicine II, Fundeni Clinical Institute, 258 Soseaua Fundeni,

Bucharest 022328, Romania

Phone: +40722235695

E-mail: laura_ate@yahoo.com thrombosis, liver congestion appears, followed by increased portal vein pressure and, ultimately, signs of liver fibrosis and liver failure.

The diagnosis is established after confirming the hepatic outflow obstruction, either by ultrasonography (US), computer tomography (CT) scan or magnetic resonance imaging (MRI) [3].

There are three morphological BCS types described, based on the site of obstruction: truncal type (consisting of obstruction of the inferior vena cava with or without obstruction of the suprahepatic veins), radicular type (consisting of obstruction of the suprahepatic veins) and venoocclusive type (consisting of the obstruction of small centrilobular veins) [4]. Imaging methods may reveal direct or indirect signs of BCS. Direct signs are: visualization of occlusion or extrinsic compression of the suprahepatic veins or inferior vena cava and stagnant or inverted venous flow and the presence of venous collaterals. Indirect signs are: hypertrophy of unaffected liver segments (most frequently the caudate lobe) and atrophy of the affected segments, which leads to the development of regeneration nodules and portal hypertension $[5]$. 


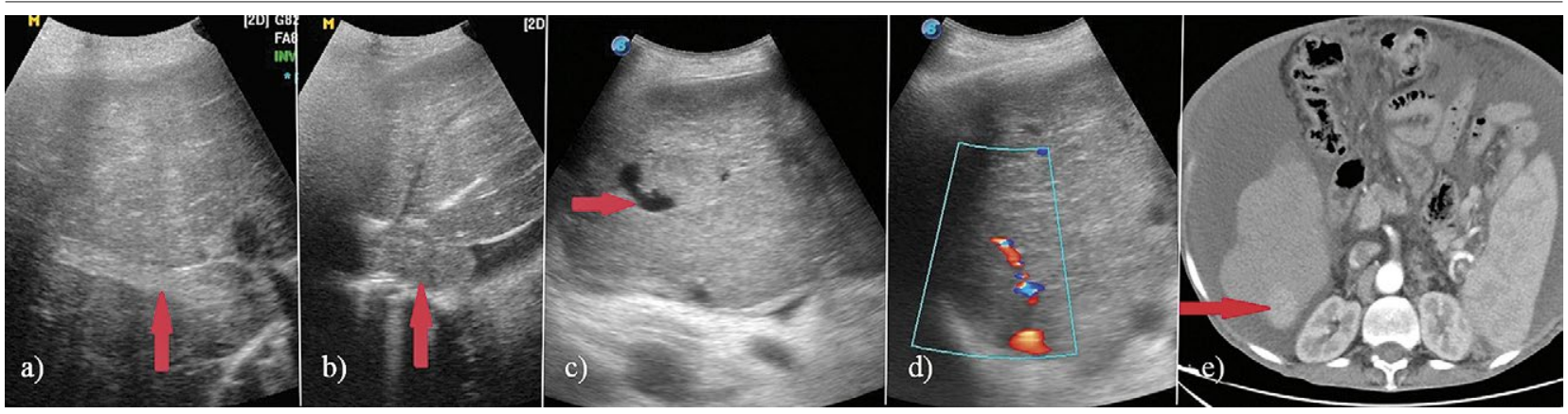

Fig 1. Abdominal ultrasonography, longitudinal subcostal view, in patient 1 showing malignant thrombosis of the right hepatic vein (arrow) (a); b) longitudinal view from right lateral position confirmed the extension of the malignant hepatic thrombus into the inferior vena cava; c) longitudinal subcostal view showed the intrahepatic collateral vascularization (arrow); d) color Doppler interrogation confirmed the narrowing and flow turbulence in the right suprahepatic vein from longitudinal subcostal view; e) abdominal CT scan revealed a nodule (arrow) in the right liver lobe with arterial enhancement suggestive for hepatocellular carcinoma.

Treatment of BCS is aimed at the underlying disease, complications of portal hypertension and the progression of thrombosis. Several options are available, including anticoagulation and Transjugular Intrahepatic Portosystemic Shunt (TIPS); the selection should be made individually for each patient [5].

We report several cases of BCS, of various etiologies and clinical evolution, emphasizing the importance of imagistic evaluation.

\section{Budd Chiari Syndrome associated with abdominal malignancy}

Case 1: A 45-year-old patient with a history of chronic hepatitis B, without prior antiviral treatment, was admitted for upper gastro-intestinal bleeding by hematemesis. Upper gastrointestinal (GI) endoscopy showed stage II esophageal varices with red spots and ligation was performed. Abdominal US revealed a focal liver lesion in the right lobe with a malignant thrombus in the right hepatic vein, extending in the inferior vena cava, with intrahepatic collateral circulation (fig $1 \mathrm{a}-\mathrm{d}$ ). Abdominal CT scan showed arterial enhancement (fig 1e) with portal wash-out of the focal liver lesion. This, in association with high levels of alpha-fetoprotein and the presence of risk factors (hepatitis B) led to the diagnosis of hepatocellular carcinoma. The patient refused any interventional procedures for the liver nodule.

Case 2: A 61-year-old asymptomatic male patient was evaluated during a routine check-up. Abdominal US revealed malignant thrombosis of the inferior vena cava (fig 2 a-c). The cause appeared to be a tumor located in the right kidney (fig 2d), with associated renal vein thrombosis (fig 2e). Abdominal CT confirmed the diagnosis of Gravitz tumor in the right kidney with venous thrombosis extending in the inferior vena cava. The patient underwent extensive surgery with right nephrectomy, resection of the thrombosed vena cava and subsequent reconstruction. The patient is now under anticoagulation therapy, with good outcome one year after surgery.
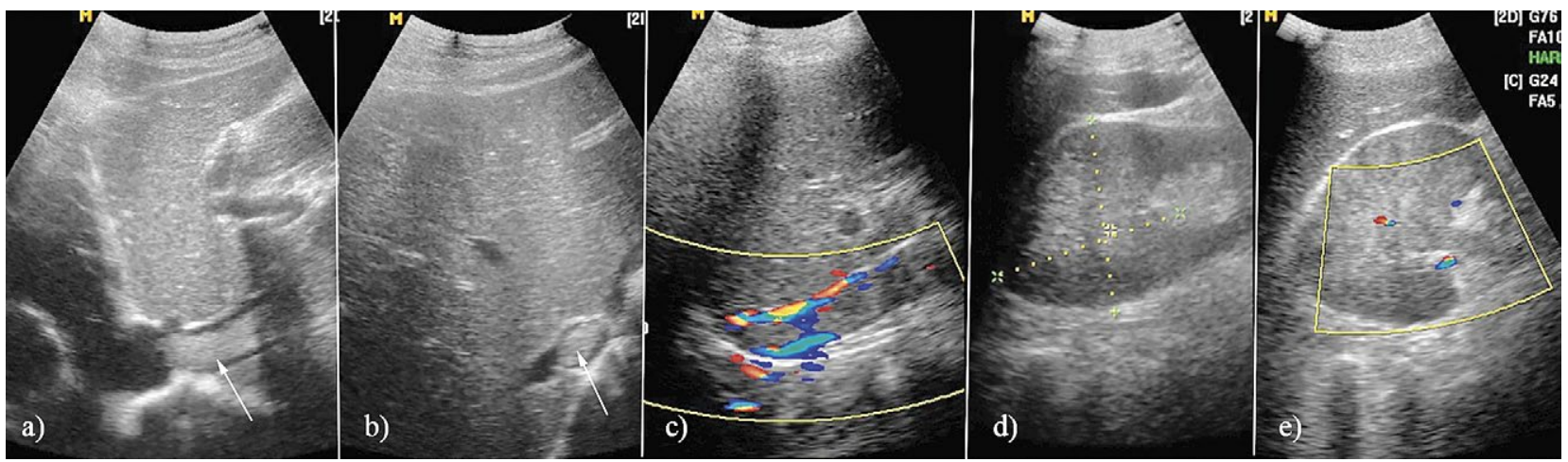

Fig 2. Abdominal ultrasonography in patient 2 showing thrombosis of the inferior vena cava in longitudinal retrohepatic section (a) and transversal section (b), confirmed by Doppler interrogation (c); d) ultrasound scan of the right kidney showing a large Gravitz tumor with renal vein thrombosis (e). 


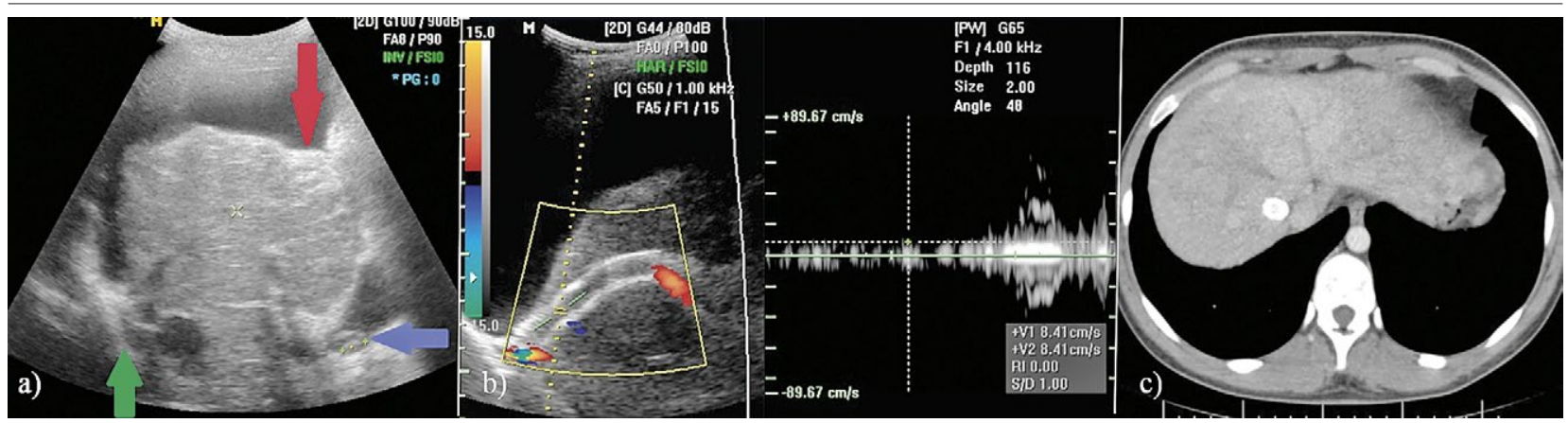

Fig 3. Abdominal ultrasonography, longitudinal scan of the right and caudate lobe, in patient 3 , with myeloproliferative disease and thrombosed TIPS: a) in greyscale, chronic alterations in the hepatic veins with two narrowed veins connecting to the inferior vena cava and threadlike aspect of the right hepatic vein (green arrow) can be observed. The caudate lobe was hypertrophied (red arrow) and the portal vein had a normal caliber (blue arrow); b) in Doppler interrogation of TIPS the absence of color Doppler signal and low velocities on pulse Doppler were found, findings suggestive for thrombosis. In the venous phase of the abdominal CT; (c) the TIPS thrombosis, narrowing of the right suprahepatic vein and perfusion lesions in the liver parenchima suggestive for ischemia were described.

\section{Budd Chiari Syndrome associated with myeloproliferative disorders}

Case 3: A 29-year-old female patient, with a history of essential thrombocythemia and hepatic vein thrombosis, having TIPS performed 5 years prior to current admission, was admitted for abdominal pain in the upper quadrants. Abdominal US revealed hypertrophy of the caudate lobe and narrowing of the hepatic veins, suggesting BCS and TIPS thrombosis. The thrombosis was confirmed by CT scan, the venous phase revealing impaired blood flow through TIPS, narrowing of the right suprahepatic vein and perfusion disorders in the liver parenchyma (liver ischemia) (fig 3). Due to the development of liver dysfunction as well as signs of portal hypertension, the patient was evaluated for liver transplantation. Echocardiography and right heart catheterization showed severe pulmonary hypertension (systolic pulmonary artery pressure of $90 \mathrm{mmHg}$ ). The patient is currently undergoing treatment with phosphodiesterase inhibitors before being listed for transplantation.

Case 4: A 30-year-old patient, with a history of liver cirrhosis of unknown etiology, with splenectomy and variceal ligation with gastric devascularization at the age of 10, was admitted for further evaluation. The patient denied alcohol abuse or use of any medication. Blood analysis showed negative viral markers, no auto-antibodies, normal serum levels of ceruloplasmin and iron. Abdominal US revealed narrowing of the right hepatic vein. The suspicion of BCS was raised. Abdominal CT scan confirmed the diagnosis, showing hypertrophy of the caudate lobe, no visualization of the suprahepatic veins and signs of portal hypertension (fig 4). Genetic determinations showed deficit of Factor V Leyden. The patient is currently undergoing anticoagulation therapy with low-molecular weight heparin with favorable out-
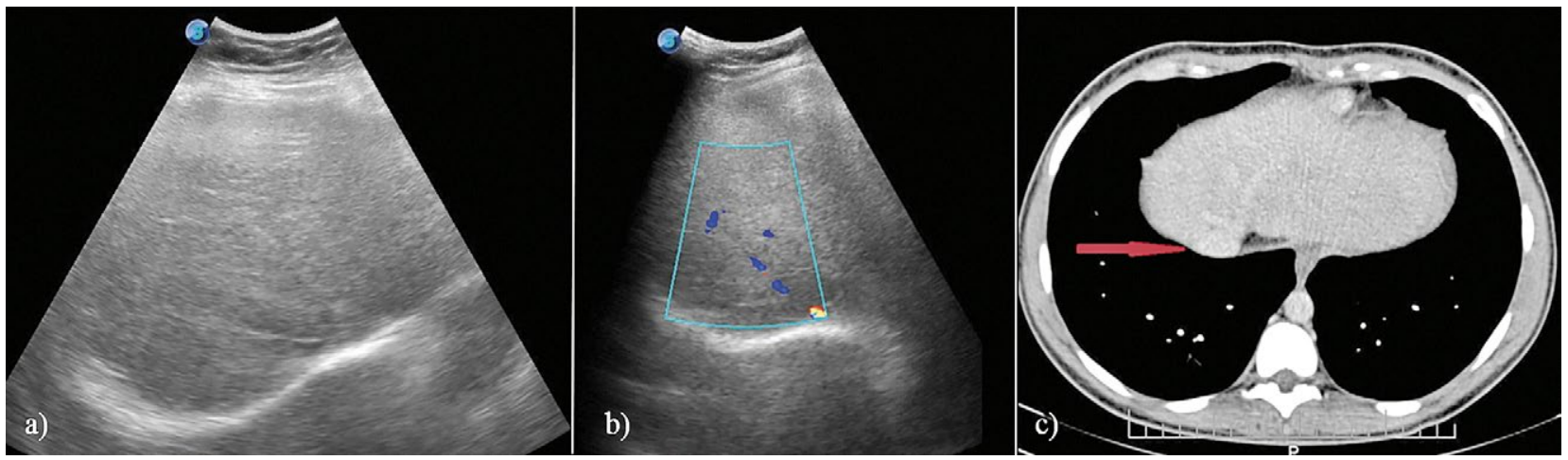

Fig 4. Abdominal ultrasonography in patient 4. Right hepatic vein with thickened wall and stenosis (a) and thread-like flow in the hepatic vein in Doppler interrogation (b) were found. Abdominal CT scan, portal phase, showing perfusion disorders in the liver parenchyma, lack of visualization of the right hepatic vein and right liver lobe hypotrophy (c). 


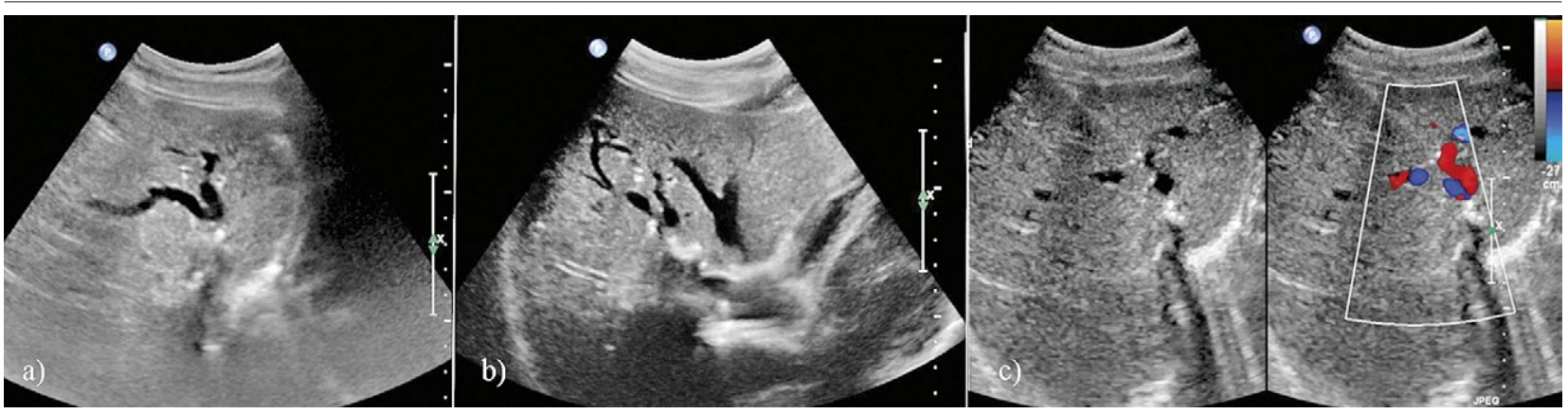

Fig 5. Abdominal ultrasound in patient 5. Oblique section of the inferior vena cava and hepatic veins, showing narrowing of the left hepatic vein (a) with retrograde venous dilatation (b). Doppler interrogation showed diminished flow in the left hepatic vein (c).

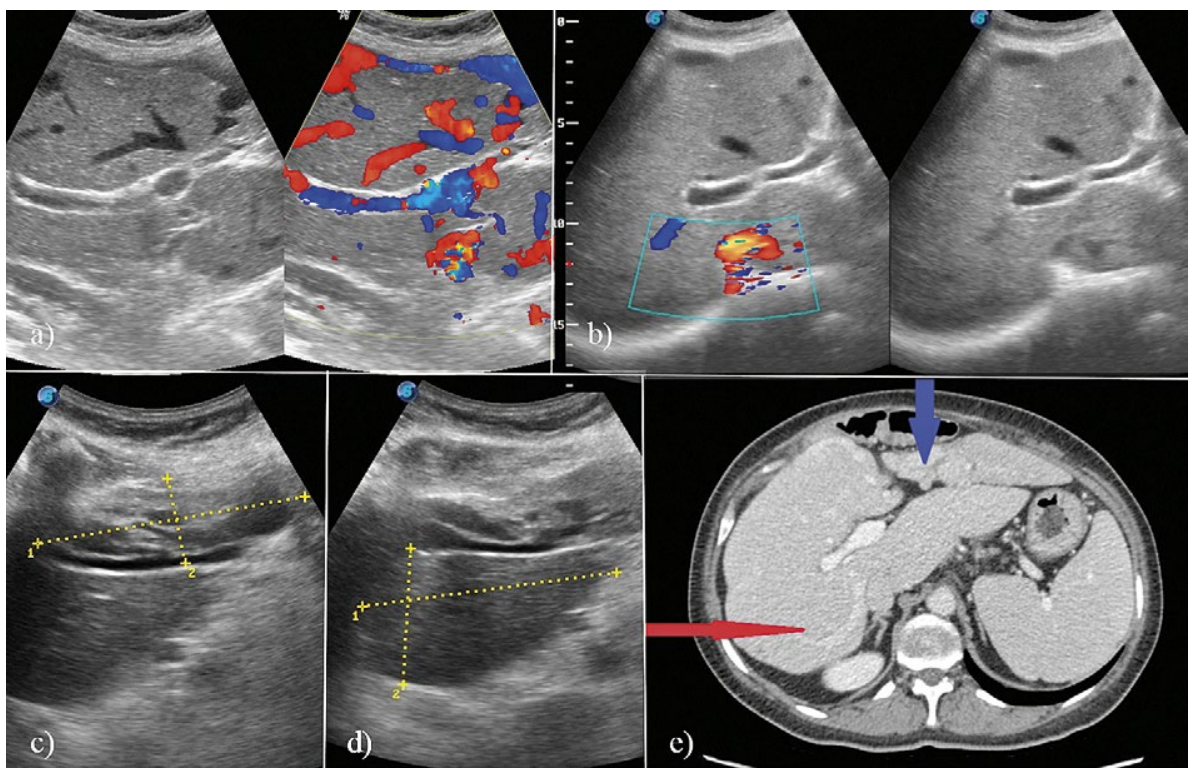

Fig 6. Abdominal ultrasonography in patient 6 . Venous collaterals are noticeable in the right liver lobe and turbulent flow in the inferior vena cava (a) due to the narrowing of the lumen (c). There is left lobe hypotrophy (c) as well as caudate lobe hypertrophy (d). Abdominal CT, venous phase, showed left lobe hypotrophy (blue arrow), caudate and right lobe hypertrophy (red arrow), distended right hepatic vein and absence of middle hepatic vein (e). come. Acenocumarol anticoagulation was not used in this case due to poor control of INR.

\section{Budd Chiari Syndrome after liver resection}

Case 5: A 45-year-old female patient, without medical complaints, with a history of multiple liver hydatid cysts for which liver resection was realized 5 years prior to current admission, was evaluated during a routine consult. Abdominal US incidentally revealed stenosis of the left hepatic vein with intrahepatic venous dilatations, permitting the diagnosis of iatrogenic BCS (fig 5). As the patient had no medical complaints and no other risk factors for thrombosis, conservative management was chosen, the patient undergoing bi-annual monitoring.

Case 6: A 53-year-old female patient, with history of liver and lung resection for hydatid cysts at the age of 29 , chronic liver disease with negative viral and auto-antibodies, was admitted for upper right quadrant abdominal pain and nausea. Clinical evaluation revealed mild hepato-splenomegaly. Blood tests were within normal limits. Abdominal ultrasound showed intrahepatic venous collaterals in the right lobe, inferior vena cava partial occlusion, and hypertrophy of the left and caudate lobe and (fig 6 a-d), findings confirmed by CT scan (fig 6 e). Secondary BCS syndrome was diagnosed. As in the previous case, due to the lack of thrombosis risk factors, the patient did not receive anticoagulation and is currently being managed conservatively, with good evolution.

In conclusion, $\mathrm{BCS}$ can be associated with a variety of medical conditions; its prompt diagnosis is critical and may change the management of these patients. The Doppler US evaluation of the hepatic veins and the careful assessment of indirect signs of hepatic obstruction are mandatory in patients with risk factors for BCS. Routine US can be successfully performed for follow-up of these patients.

Conflict of interest: none 


\section{References}

1. Darwish Murad S, Plessier A, Hernandez-Guerra M, et al. Etiology, management, and outcome of the BuddChiari syndrome. Ann Intern Med 2009;151:167175.

2. Aydinli M, Bayraktar Y. Budd-Chiari syndrome: Etiology, pathogenesis and diagnosis. World J Gastroenterol 2007;13:2693-2696.
3. Grus T, Lambert L, Grusová G, Banerjee R, Burgetová A. Budd-Chiari Syndrome. Prague Med Rep. 2017;118:69-80.

4. Khan F, Armstrong MJ, Mehrzad H, et al. Review article: a multidisciplinary approach to the diagnosis and management of Budd-Chiari syndrome. Aliment Pharmacol Ther 2019;49:840-863.

5. European Association for the Study of the Liver. EASL Clinical Practice Guidelines: Vascular diseases of the liver. J Hepatol 2016;64:179-202. 